\title{
Alocação de canais para redes veiculares baseada no comportamento do nó
}

\author{
Roni Francis Shigueta ${ }^{1}$, Marcelo Pellenz ${ }^{1}$ and Mauro Fonseca ${ }^{2}$ \\ ${ }^{1}$ Pontifícia Universidade Católica do Paraná - PUCPR and ${ }^{2}$ UTFPR \\ *shigueta@yahoo.com; marcelo@ppgia.pucpr.br; mauro.fonseca@gmail.com.br \\ Recebido: 08/03/2019. Revisado: 23/04/2019. Aceito: 22/05/2019.
}

\begin{abstract}
Resumo
Este trabalho apresenta uma estratégia de alocação de canal baseada no comportamento do nó para redes veiculares (VANETs). A principal contribuição deste trabalho é considerar algumas características do comportamento do nó para guiar o processo de alocação de canais em VANETs. No trabalho é usada uma abordagem distribuída que permite a estratégia rapidamente responder a mudanças de topologia na rede, limitando a troca de mensagens. Os canais alocados são do espectro do padrão IEEE 802.11p e as métricas de avaliação são: vazão agregada, taxa de entrega de pacotes, atraso fim a fim e overhead. Os resultados mostram que a estratégia apresenta mais alta vazão e taxa de entrega de pacotes com menor atraso fim a fim quando comparado aos métodos de alocação de canais randômico e simples. Quando comparado a uma abordagem centralizada, a estratégia apresenta desempenho próximo ao do algoritmo Tabu, mas com menor overhead.
\end{abstract}

Palavras-Chave: Alocação de Canal; Comportamento do Nó; IEEE 802.11p; Redes Veiculares.

\begin{abstract}
This work presents a node behavior-based channel allocation strategy for Vehicular Ad hoc NETworks (VANETs). The main contribution of this work is to consider some characteristics of the node behavior to guide the channel allocation process in VANETs. In the work is used a distributed approach that allows the strategy quickly to respond to changes in the network topology while limiting the exchange of messages. The channels allocated are of the spectrum of the IEEE 802.11p standard and the evaluation metrics are: throughput, packet delivery rate, end-to-end delay, and overhead. The results show that the strategy presents higher aggregated throughput and packet delivery rate, with lower end-to-end delay when compared to random channel allocation method and single channel. When compared to a centralized approach, the strategy presents performance closer to Tabu algorithm, but with lower overhead.
\end{abstract}

Key words: Channel Allocation; IEEE 802.11p; Node Behavior; Vehicular Networks.

\section{Introdução}

Com o avanço da indústria automotiva, cada vez mais veículos estão sendo equipados com sensores e equipamentos que permitem a comunicação entre veículos para evitar colisões, relatar acidentes, áreas congestionadas ou para acessar algum tipo de serviço disponível na rede veicular. A comunicação entre veículos consome recursos tais como processamento, memória, energia e canal de freqüência. A disponibilidade de processamento, memória e energia pode ser aumentada utilizando mais processadores, memórias ou mais fontes de energia, porém o canal de freqüência não é um recurso facilmente escalável. Ele é um recurso regulamentado por agências governamentais e está se tornando cada vez mais escasso devido à grande quantidade de dispositivos competindo pelo seu uso. Neste contexto, este trabalho apresenta uma estratégia de alocação de 
canais baseada no comportamento do nó para redes veiculares. No trabalho é utilizada uma representação de grafo em que cada nó representa um veículo e cada aresta do grafo representa um enlace de comunicação entre veículos. Para guiar o processo de alocação de canais são considerados como comportamento do nó: o tráfego gerado pelo nó, sua mobilidade e sua popularidade. Para permitir uma rápida resposta a mudança de topologia da rede é utilizada uma abordagem distribuída limitando a troca de mensagens a 2 saltos de distância. Para a validação, a estratégia é comparada com 3 outros métodos: alocação centralizada, randômica e de canal simples e os resultados são avaliados em termos de vazão agregada, taxa de entrega de pacotes, atraso fim a fim e overhead.

Este artigo é organizado da seguinte forma: a Seção 2 apresenta a arquitetura de uma rede veicular. A Seção 3 aborda os trabalhos relacionados de alocação de canais em VANETs. A Seção 4 apresenta os modelos de canal, interferência e de rede. A Seção 5 detalha o mecanismo de alocação de canais. A Seção 6 apresenta o cenário e a avaliação da performance da estratégia. Por fim, a Seção 7 conclui o artigo.

\section{Arquitetura de uma rede veicular}

Uma VANET é uma rede veicular que tem uma arquitetura composta de 3 componentes (Hande and Muddana; 2016): RSU (Road Side Unit), OBU (On Board Unit) e AU (Application Unit). A RSU é um dispositivo fixado ao longo da rua, avenida ou rodovia ou em pontos específicos equipados com algum dispositivo de rede. A RSU permite estender a área de comunicação distribuindo informações para veículos que estão localizados em posições mais remotas. Ela também permite a comunicação com outras RSUs, a Internet ou algum tipo de serviço em nuvem. A OBU é um dispositivo instalado dentro do veículo, usado para a troca de informações com as RSUs ou outras OBUs. A AU é um dispositivo dentro do veículo que executa as aplicações e usa a capacidade de comunicação da OBU.

Dependendo do tipo de comunicação entre RSUs e OBUs, as VANETs podem ser classificadas como V2V (Vehicle to Vehicle), V2I (Vehicle to Infrastructure) e híbrida (Chen; 2015). No caso V2V, a comunicação ocorre entre as OBUs dos veículos, de maneira autônoma, sem a intervenção de um coordenador de rede. Este tipo de comunicação também é conhecida como Ad hoc. Em V2I a comunicação ocorre entre a OBU de um veículo e a RSU. Este tipo de comunicação utiliza uma infraestrutura (no caso a RSU) para propagar as informações por distâncias maiores ou para a Internet. Por fim, no modo de comunicação híbrido a comunicação pode ocorrer tanto entre as OBUs quanto com as RSUs. A Fig. 1 mostra as formas de comunicação em VANETs. Na figura é possível observar que um veículo pode se comunicar com outros veículos e com a RSU e que as RSUs podem se comunicar entre si para o encaminhamento de mensagens.

\section{Trabalhos relacionados}

Na literatura, vários autores propõem a alocação de canais para redes veiculares.

Em Zhao et al. (2014), os autores apresentam um mecanismo de alocação de canais que seleciona os canais com menor uso, porém não consideram a mobilidade e o tráfego no processo de alocação de canais.

Em Hussain et al. (2017), os autores apresentam um esquema de acesso ao canal centralizado para redes veiculares, considerando o tempo que o veículo leva para sair do alcance da RSU. O esquema prioriza a mobilidade e serviços de emergências no processo de alocação de canal, mas não considera o tráfego na rede.

Em Gupta et al. (2016), os autores apresentam um esquema cognitivo de alocação de canal dinâmico na banda adjacente da faixa de frequência DSRC (Dedicated Short-Range Communication). O esquema somente usa a ocupação de canal como critério de alocação de canal.

Em Nguyen et al. (2015) é apresentado um mecanismo de alocação de canais para VANETs usando TDMA. O esquema é baseado em cluster onde os membros do cluster solicitam um intervalo de tempo de transmissão para o coordenador do cluster. Neste mecanismo, os veículos não tem prioridade e os canais são alocados de acordo com a requisição de intervalo de tempo de alocação.

Em Tabassum et al. (2016) é proposto um mecanismo de alocação de canais para redes veiculares cognitivas que representa a rede como um grafo de conflito e formula o problema de alocação de canais como um problema de Programação Linear Inteira Mista. O mecanismo não considera outros parâmetros como tráfego e mobilidade na alocação de canais.

Em Chu et al. (2012), os autores propõe um esquema de atribuição de canais distribuido (DCAS) usando o padrão IEEE 802.11p considerando um cenário de autoestrada. DCAS é baseado em TDMA e para evitar a interferência entre os enlaces são selecionados diferentes intervalos de tempo de utilização para os enlaces a dois saltos e a prioridade de alocação de canais é somente para mensagens de emergência.

Em Park et al. (2013) é apresentado um mecanismo de alocação de canais de serviços dinâmicos para redes veiculares. O objetivo é atribuir um canal de serviço (SCH) para cada veículo usando categorias de acesso para melhorar a vazão na rede. O mecanismo usa o histórico das mensagens de anunciamento de serviços enviadas pelos veículos e seleciona o canal com maior capacidade, mas ele não prioriza o nó que irá usar o canal.

Em Reddy and Reddy (2017), os autores propõem um esquema de alocação de canais baseado na posição do nó para VANETs. No esquema, a rede é dividida em regiões chamada de clusters. Cada cluster seleciona um nó como coordenador da sua região e ele é responsável por alocar um intervalo de tempo de uso (baseado em TDMA) para cada nó dentro do cluster. No esquema, veículos que estão mais próximos do centro do cluster obtêm mais largura de banda do que os veículos que estão na extremidade. Embora o esquema usa a mobilidade do nó, ele não considera o 


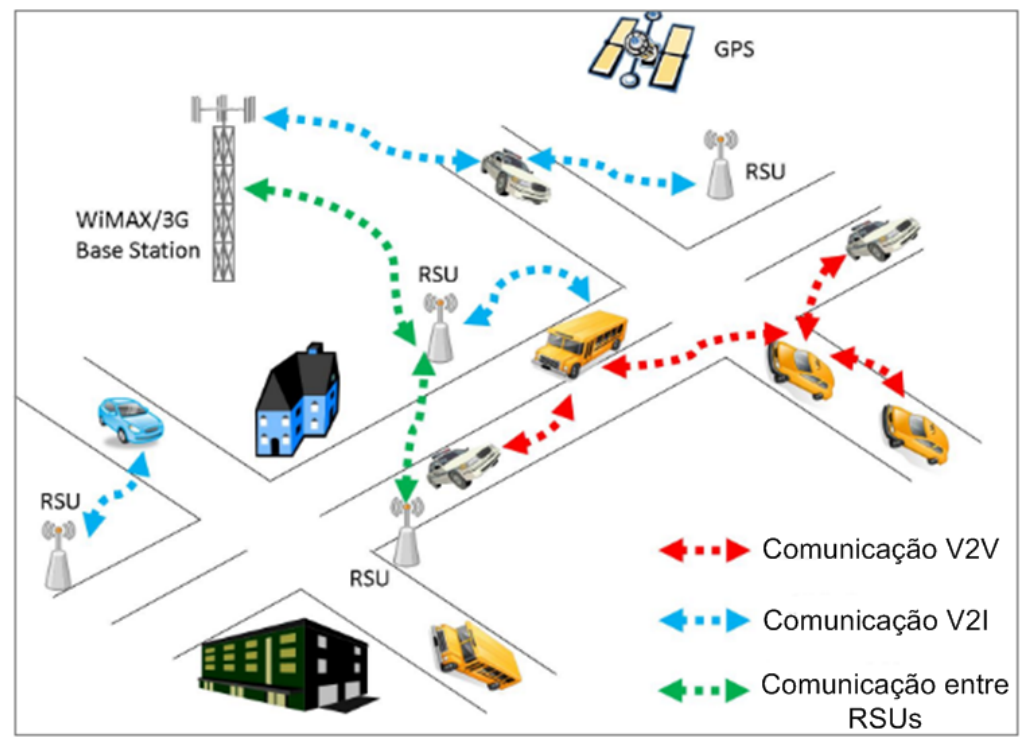

Figura 1: Formas de comunicação em VANETs (Lemos; 2010)

tráfego como critério de alocação de canal.

Em Peng et al. (2014) é apresentado um esquema de alocação multi-canal baseado em cluster usando o espectro do padrão IEEE 802.11p. Na comunicação dentro do cluster (intra-cluster) é usado um esquema não competitivo baseado em TDMA, que seleciona um intervalo de tempo disponível para a OBU.

Em Tomar and Verma (2010) é proposto um protocolo de alocação de canais centrado na RSU. O objetivo é minimizar o tempo de alocação de canais e o overhead. A RSU divide a região em clusters e o canal em cada cluster é dividido em intervalos de tempo de uso. Um intervalo de tempo é alocado para um veículo considerando somente a prioridade do tipo de serviço e a disponibilidade do canal.

Em Frigau (2015), os autores apresentam um mecanismo de alocação de canais que seleciona oportunisticamente um canal de serviço entre os seis canais disponíveis no padrão IEEE 802.11p. A seleção de canal é baseada somente na ocupação do canal e não considera outros parâmetros e a prioridade do nó.

Em Mahdi et al. (2016), os autores apresentam um protocolo para OBU baseado em mobilidade, onde a mudança de canal devido a mobilidade espacial ocorre quando um nó entra ou deixa a rede. No trabalho, outros parâmetros como tráfego ou prioridade do nó não são considerados.

Em Sulistyo and Alam (2018), os autores apresentam um estudo de alocação multicanal utilizando acesso múltiplo de freqüência ortogonal em VANETs, considerando o modelo físico de canal. Para otimizar a alocação de canais, os autores utilizam um método de otimização baseado em bactérias de modo a incrementar a relação sinal-ruído mais interferência (SINR) e a vazão da rede.

Nossa proposta difere dos trabalhos relacionados na literatura porque utiliza vários parâmetros como tráfego, mobilidade e popularidade do nó, simultaneamente, para guiar o processo de alocação de canais.

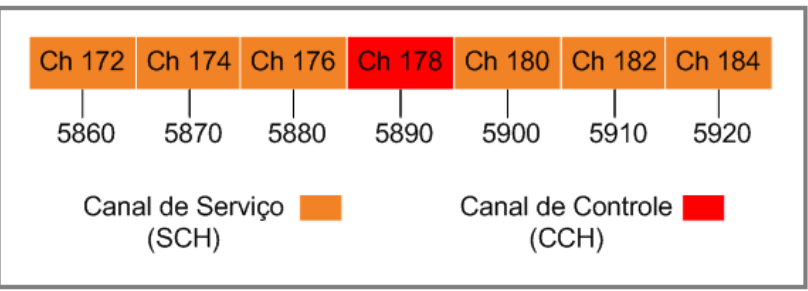

Figura 2: Canais do padrão IEEE 802.11p

\section{Modelos de Canal, Interferência e de Rede}

Esta seção apresenta os modelos de canal, interferência e de rede utilizados neste trabalho.

\subsection{Modelo de Canal}

O modelo de canal utilizado é baseado no padrão IEEE 802.11p (Bharati and Zhuang; 2018). Neste padrão, o espectro de $75 \mathrm{MHz}$ (entre $5.850 \mathrm{GHz}$ e $5.925 \mathrm{GHz}$ ) é dividido em sete canais (do número 172 ao 184) (Lusheng et al.; 2012). A capacidade de cada canal é de $10 \mathrm{MHz}$. O canal 178 é o canal de controle (CCH) e é reservado para comunicações de segurança. Os outros seis canais são canais de serviço (SCH) e podem ser utilizados para aplicações que envolvam ou não segurança. Aplicações seguras representam serviços de alta prioridade como mensagens de alerta de colisões ou acidentes. Aplicações não seguras consiste em serviços que não implicam em risco, como por exemplo, acesso a Internet ou acesso a serviços de vídeo. Neste trabalho é usado o canal 172 para a troca de mensagens de controle, assim, as mensagens do mecanismo de alocação de canais não concorrem com os serviços de mensagens de segurança do canal 178. A Fig. 2 ilustra os canais de controle e de serviços do padrão IEEE 802.11p. As frequências dos canais estão expressas em $\mathrm{MHz}$. 


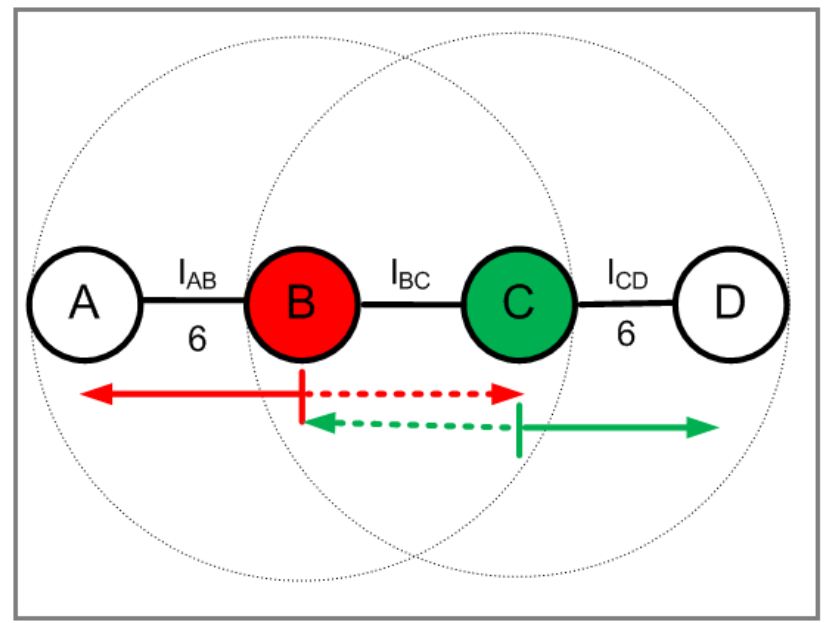

Figura 3: Interferência entre enlaces

\subsection{Modelo de Interferência}

Neste trabalho é considerado o modelo de interferência a 2 saltos (Subramanian et al.; 2008). Neste modelo, dois enlaces são considerados interferentes se eles estão a 2 saltos de distância entre si e utilizam o mesmo canal. Este modelo pode ser usado para predizer se um canal alocado para um enlace irá interferir ou não nos vizinhos a dois saltos. A Fig. 3 mostra um exemplo da interferência entre enlaces no modelo de interferência a 2 saltos.

No exemplo, o nó $\mathrm{B}$ envia dados para o nó $\mathrm{A}$ (através do enlace $l_{A B}$ ) usando o canal 6. Quando o nó $B$ envia dados para o nó $A$, o sinal também se propaga na direção do nó $\mathrm{C}$ (seta vermelha tracejada a direita) e ele interfere na transmissão do node $\mathrm{C}$ com o nó $\mathrm{D}$ (enlace $\mathrm{l}_{C D}$ ) devido ao uso do mesmo canal que o link $l_{A B}$. Na transmissão entre o nó $C$ e o nó $D$, o sinal também se propaga em direção do nó $\mathrm{B}$ (seta verde tracejada a esquerda), causando interferência na transmissão do nó $B$. Na Fig. 3, o link $1_{B C}$ é um vizinho a 1 salto do link $\mathrm{l}_{A B}$ e o link $\mathrm{l}_{C D}$ é um vizinho a 2 saltos do link $l_{A B}$.

No trabalho é considerado o modelo de interferência a 2 saltos devido aos outros modelos medirem somente a interferência a 1 salto. 0 modelo de interferência a 2 saltos é capaz de estimar as interferências geradas pelos vizinhos dos nós que estão recebendo pacotes (vizinhos a 2 saltos). O uso do modelo de interferência a 2 saltos permite definir uma métrica de qualidade de canal (QC), baseada no número de vizinhos a 2 saltos que usam o mesmo canal. Esta métrica é dada por (Sallem et al.; 2012):

$$
Q C=1 /\left(1+I_{i, c}\right)
$$

onde: $I_{i}, c$ é o número de vizinhos a 2 saltos de um nó $i$ usando o canal $c$.

Um menor número de ocorrências de vizinhos a 2 saltos usando o mesmo canal resulta em um menor nível de interferência e uma qualidade de canal maior.

\subsection{Modelo de Rede}

O modelo de rede considerado é de uma rede veicular urbana. No modelo, a rede é representada por um grafo, onde os nós representam os veículos e as arestas os enlaces de comunicação entre os veículos. O modelo utiliza duas interfaces: uma para manter a conectividade mínima entre os nós, usando um canal de controle comum (CCC) e a outra para enviar e receber dados. Estas duas interfaces estão localizadas na OBU do veículo. A interface de controle CCC, opera no canal 172 permitindo a troca de mensagens de canais disponíveis, mensagens de atribuição de canais, mensagens de requisição de comutação de canais (RTS/CTS) (Rocha da Silva and Rezende; 2006) e mensagens de roteamento. A segunda interface é usada para transmitir dados e comuta de canal de acordo com o algoritmo de alocação de canal.

\section{0 mecanismo de alocação de canais}

O mecanismo de alocação de canais é representado como uma máquina de estados composta por 4 estados: 1) Escalonador, 2) Gerente de Topologia, 3) Mecanismo de Alocação, 4) Mecanismo de Interação.

A seguir é detalhado cada um dos estados.

\subsection{Estado1 (Escalonador)}

O escalonador é responsável por enviar mensagens de Hello e mensagens de Interação. As mensagens de Hello são enviadas periodicamente e permite um nó trocar informações sobre canais disponíveis, mobilidade (posição, velocidade, e direção) e notificar sua vizinhança que ele está ativo. As informações de mobilidade são usadas para calcular a posição futura de um nó. Esta informação é usada como um parâmetro para guiar o processo de alocação de canais.

Além do envio das mensagens de Hello, o escalonador envia mensagens de Interação. Estas mensagens são enviadas aos nós vizinhos, com o objetivo de notificar os canais que foram alocados pelo nó. Um nó ao receber uma mensagem de Interação pode aceitar ou rejeitar a alocação de canal de acordo com a sua prioridade como irá ser visto no Estado4 (Mecanismo de Interação).

\subsection{Estado2 (Gerente de Topologia)}

O Gerente de Topologia é responsável por verificar a estabilidade da topologia da rede e criar uma lista de canais que podem ser alocados para os enlaces de um nó. A topologia é considerada estável quando ela não muda depois de duas trocas consecutivas de mensagens de Hello entre um nó e seus vizinhos. Se a topologia é instável, o nó permanece no Estado2, recebendo as mensagens de Hello, até que a condição de estabilidade seja encontrada. Assim, o processo de alocação de canais somente inicia quando todos os nós vizinhos são detectados.

Quando um nó recebe uma mensagem de Hello, ele extrai os canais da mensagem e armazena eles em uma lista de canais disponíveis. A intersecção entre a lista de canais disponíveis e a lista de canais 


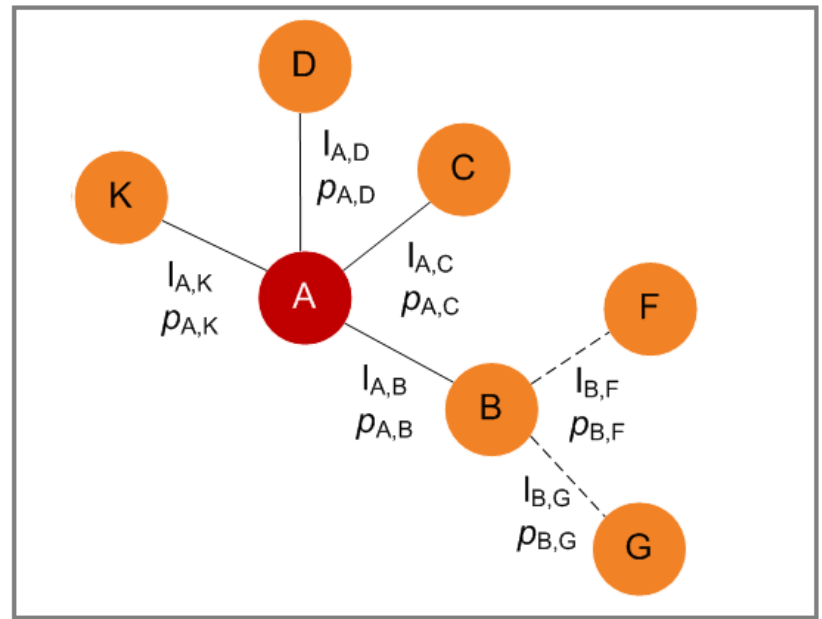

Figura 4: Exemplo de grafo de rede

locais resulta em uma lista de canais comuns. Esta lista contém os canais que são comuns a um nó e seus vizinhos e que estão disponíveis para serem selecionados pelo algoritmo de alocação de canais.

\subsection{Estado3 (Mecanismo de Alocação)}

O Estado3 é executado sempre que um nó é inicializado ou quando a topologia da rede muda e se torna estável. Neste estado, o mecanismo de alocação de canais é executado baseado no conhecimento local do nó. A Fig. 4 mostra um exemplo de um grafo de rede em que um nó $A$ possui 4 links $\left(l_{A, B}, l_{A, C}, l_{A, D}\right.$, $\left.1_{\mathrm{A}, \mathrm{K}}\right)$.

Os nós na vizinhança do nó A (a 1 salto) possuem características que representam o comportamento do nó, tais como mobilidade, tráfego e popularidade. Estas características são associadas a uma função de comportamento de enlace que é usada para definir a prioridade do enlace. Assim, o nó A tem uma função de prioridade de enlace $\left(p_{\mathrm{A}, \mathrm{B}}, p_{\mathrm{A}, \mathrm{C}}, p_{\mathrm{A}, \mathrm{D}}, p_{\mathrm{A}, \mathrm{K}}\right)$ para cada enlace associado a um nó vizinho.

A função de prioridade de enlace para um nó A com um nó vizinho K é dada por:

$$
p_{\mathrm{A}, \mathrm{K}}(t+\Delta)=\left\{\begin{array}{ccc}
0 & \text { se } & \left|d_{\mathrm{A}, \mathrm{K}}(t+\Delta)\right|>r_{\mathrm{m}} \\
b_{\mathrm{A}, \mathrm{K}}(t+\Delta), & \text { se } & \left|d_{\mathrm{A}, \mathrm{K}}(t+\Delta)\right| \leq r_{\mathrm{m}}
\end{array}\right.
$$

onde:

$t$ é o tempo corrente e $\Delta$ é a janela de tempo futura. $r_{m}$ é o raio de transmissão máximo do nó.

$d_{\mathrm{A}, \mathrm{K}}(t+\Delta)$ é a distância estimada, no tempo $t+\Delta$, entre o nó local $\mathrm{A}$ e o nó vizinho $\mathrm{K}$ que pertence ao enlace $l_{A, K}$.

$b_{A, K}(t+\Delta)$ é a função de comportamento do enlace $l_{\mathrm{A}, \mathrm{K}}$ no tempo $t+\Delta$ e é dada por:

$$
b_{\mathrm{A}, \mathrm{K}}(t+\Delta)=\alpha *\left(1-M_{\mathrm{A}, \mathrm{K}}(t+\Delta)\right)+\beta * \mathrm{~T}_{\mathrm{A}, \mathrm{K}}(t)+\gamma * \mathrm{D}_{\mathrm{K}}(t)
$$

onde:

$\alpha, \beta, \gamma$ são pesos aplicados para cada parâmetro de comportamento e depende do cenário da rede.
$M_{\mathrm{A}, \mathrm{K}}(t+\Delta)$ é o parâmetro normalizado que representa a estimativa da distância entre o nó $\mathrm{A}$ e o nó vizinho $K$, no tempo $t+\Delta$.

$T_{A, K}(t)$ é o parâmetro normalizado que representa o tráfego no link $\mathrm{l}_{\mathrm{A}, \mathrm{K}}$ no tempo $t$.

$D_{\mathrm{K}}(t)$ é o parâmetro normalizado que representa a popularidade do nó $\mathrm{K}$, no tempo $t$. Este parâmetro corresponde ao grau do nó e representa o número de vizinhos que o nó possui. Nós consideramos este parâmetro devido a tendência dos nós mais populares atraírem outros nós.

Na equação (3), o valor da função comportamento aumenta com o decréscimo do valor do parâmetro de mobilidade e o aumento do valor do tráfego e do grau do nó (popularidade).

Depois de calcular a prioridade de todos os enlaces de um nó, eles são inversamente ordenados de acordo com sua prioridade. Os enlaces com maior prioridade irão receber canais com mais alta qualidade, calculados de acordo com a equação (1).

Além de realizar a alocação de canais, um nó calcula sua prioridade. A prioridade do nó é usada para atribuir uma ordem de aceitação de alocação de canais entre nós vizinhos. A prioridade para um nó $A$, considerando $n$ enlaces é calculada por:

$$
P_{\mathrm{A}}(t+\Delta)=\frac{\sum_{i=1}^{n} p_{\mathrm{A}, i}(t+\Delta)}{n} \text { s.a. } n>0
$$

onde:

$p_{\mathrm{A}, i}(t+\Delta)$ é a prioridade do enlace $\mathrm{l}_{\mathrm{A}, i}$ no tempo $t+\Delta$, dada pela equação (2).

A alocação de canal em cada enlace e o valor da prioridade do nó são enviadas aos nós vizinhos utilizando mensagens de Interação.

\subsection{Estado4 (Mecanismo de Interação)}

Quando um nó recebe uma mensagem de Interação, ele primeiro compara a prioridade do nó vizinho com sua prioridade local. Se a prioridade da mensagem recebida é menor ou igual a prioridade do nó local, a mensagem é descartada. Caso contrário, o nó aceita a alocação de canal proposta pelo nó vizinho para o enlace que recebeu a mensagem e ele recalcula a alocação de canais para seus outros enlaces (procurando por um canal com o menor número de ocorrências de alocação). A Fig. 5 mostra o nó B que ao receber uma mensagem de Interação de maior prioridade do nó A, recalcula os canais para os demais nós.

A alocação de canal ocorre na sequencia em que os enlaces foram ordenados, de acordo com a prioridade de cada enlace. A Fig. 6 mostra o algoritmo de alocação de canal para os nós com menor prioridade.

No algoritmo, para cada enlace ordenado $i$ da lista de enlaces L (linha1), se ele é o enlace que recebeu a mensagem de Interação (linha 3), ele aceita o canal proposto pelo nó vizinho (linha 5). Caso contrário (linha 6), o algoritmo seleciona o canal com menor número de ocorrências em InterferingChannelList (linha 9). InterferingChannelList contém os canais interferentes a 2 saltos. $O$ enlace e o canal são atribuídos as listas AssignedLinkList (linha 13) e AssignedChannelList (linha 14), respectivamente. A 


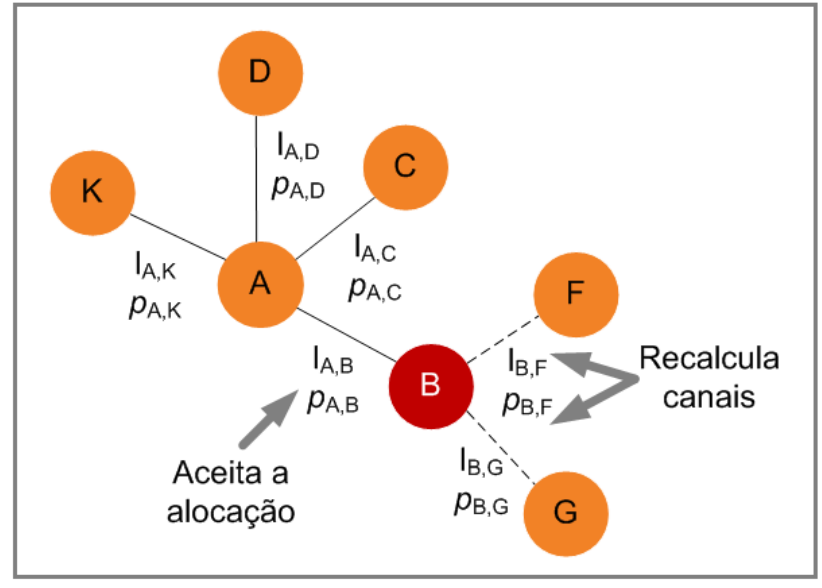

Figura 5: Recalculo de canais no nó de menor prioridade

troca das mensagens de Interação é limitada a 2 saltos.

\section{Cenário e Avaliação de Performance}

Nesta seção é descrita as simulações realizadas usando o simulador NS-v3 (nsnam.org; 2016) considerando o cenário Manhattan Grid. Neste cenário, os nós podem se mover nas vias horizontais ou verticais. Na intersecção de uma via horizontal e vertical, o nó pode virar a esquerda, a direita ou seguir em frente de acordo com uma probabilidade. Nas simulações é considerado o modelo apresentado em Aschenbruck et al. (2016). Este modelo apresenta 3 parâmetros extras, permitindo definir a velocidade, a probabilidade de pausa e tempo máximo de pausa dos nós.

\subsection{Cenário de avaliação}

O cenário de avaliação corresponde a uma rede constituída de 40 veículos distribuídos em uma área de $500 m$ x 500m com uma faixa de transmissão de $100 \mathrm{~m}$. A topologia da estrada é composta por 6 blocos nos eixos x e y do plano cartesiano. Os valores da velocidade mínima, probabilidade de pausa e tempo máximo de pausa são de $0 \mathrm{~m} / \mathrm{s}, 0,3$ e 30 segundos, respectivamente. A velocidade dos veículos variam

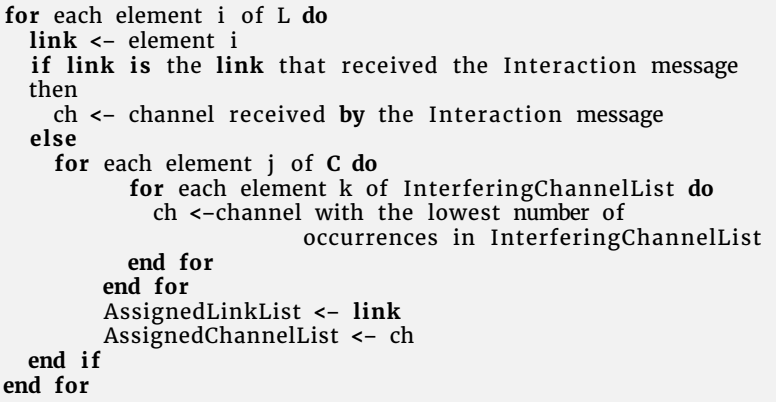

Figura 6: Algoritmo de Alocação de Canal de 0 a $5 \mathrm{~m} / \mathrm{s}$. A probabilidade do veículo mudar de direção é 0.3. A distribuição e mobilidade dos veículos são determinadas pelo modelo de mobilidade Manhattan grid. Na simulação, 4 fluxos de dados foram gerados (cada um com 3000 pacotes) com o par de origem e destino gerados randomicamente, seguindo uma distribuição uniforme. O tamanho do pacote usado foi de 1000 bytes e o tempo de simulação foi de 72 segundos. Foram executadas 1000 simulações e os resultados foram mostrados com intervalo de confiança de $95 \%$.

\subsection{Comparações entre os métodos de alocação de canais}

A estratégia proposta é comparada com 3 outros métodos: alocação de canal centralizada (TABU) (Subramanian et al.; 2008), alocação de canal randômica (RANDOM), e alocação de canal simples (SC). Em VANETs, o método RANDOM é largamente utilizado pelas RSUs e OBUs para anunciar seus serviços, não utilizando um mecanismo eficiente de alocação de canais. Em nossa implementação de RANDOM, é utilizada o mesmo mecanismo (da estratégia proposta) para notificar os nós vizinhos quais canais deverão ser utilizados. Dessa forma, no método de alocação RANDOM, os nós de origem e destino são sincronizados no mesmo canal que foi randomicamente selecionado (evitando que o nó de origem escolha um canal e o de destino um outro canal).

\subsection{Métricas de avaliação}

Para avaliar o desempenho da estratégia proposta, são utilizadas 4 métricas: (1) vazão agregada, que é a soma de pacotes entregues para todos os nós da rede por unidade de tempo, (2) taxa de entrega de pacotes, que é o número de pacotes recebidos em relação ao número de pacotes enviados, (3) atraso fim a fim, que é o intervalo de tempo que um pacote leva para encontrar o seu destino, e (4) overhead, que é o número de bytes trafegado no canal de controle.

\subsection{Resultados da simulação}

A Fig. 7 ilustra a vazão agregada em função do número de canais.

A estratégia proposta apresenta resultados próximos da abordagem centralizada, tendo de $4,4 \%$ a $2,98 \%$ de decréscimo de performance quando o número de canais varia entre 2 e 4 . O impacto da estratégia proposta e TABU é linear para 2,3 e 4 canais e quando comparado com RANDOM o ganho observado pela estratégia proposta é entre $9,2 \%$ a $12,61 \%$ e de $21,15 \%$ a $28,25 \%$ quando comparado a SC. Acima de 4 canais ocorre a saturação da vazão agregada para a estratégia proposta e TABU, pois todos os enlaces a 2 saltos estão usando um canal diferente. Como esperado, o desempenho do método SC não é impactado pelo número de canais.

A Fig. 8 mostra a taxa de entrega de pacotes média.

A estratégia proposta apresenta valores de taxa de entrega de pacotes média muito próximos de TABU com um decréscimo de $2,9 \%$. Comparado a RANDOM, 


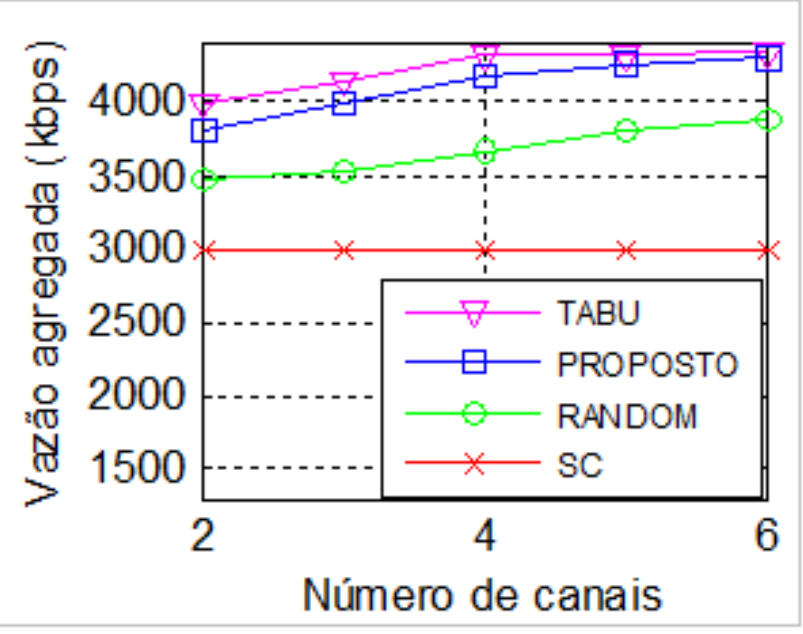

Figura 7: Vazão agregada

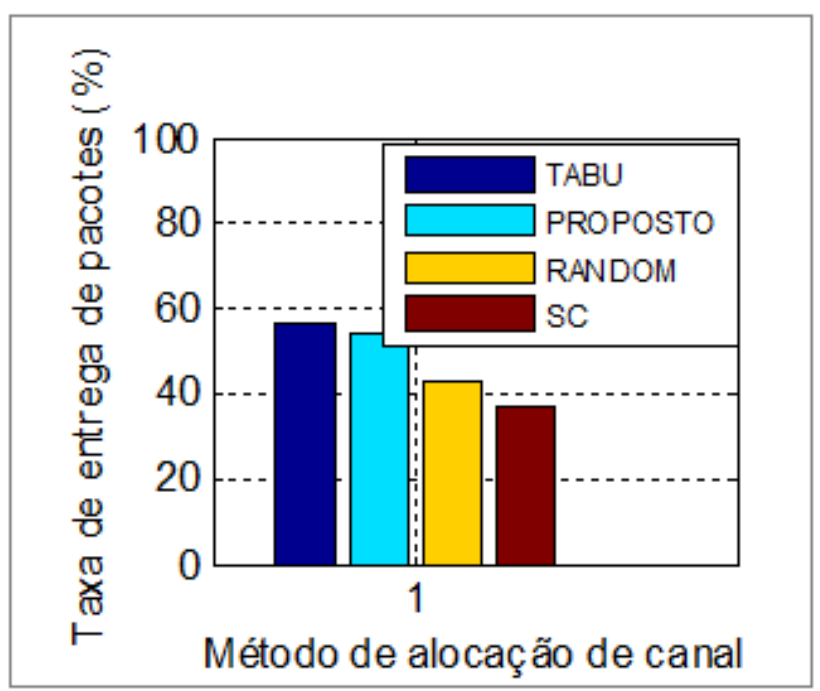

Figura 8: Taxa de entrega de pacotes média

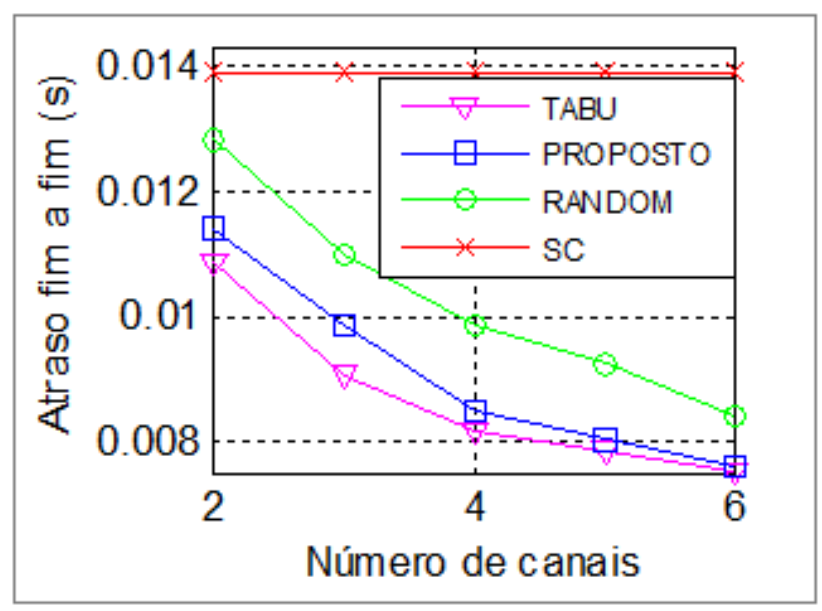

Figura 9: Atraso fim a fim

o ganho é de aproximadamente $11,65 \%$ e de $17,18 \%$ quando comparado a SC.

A Fig. 9 apresenta $o$ atraso fim a fim.

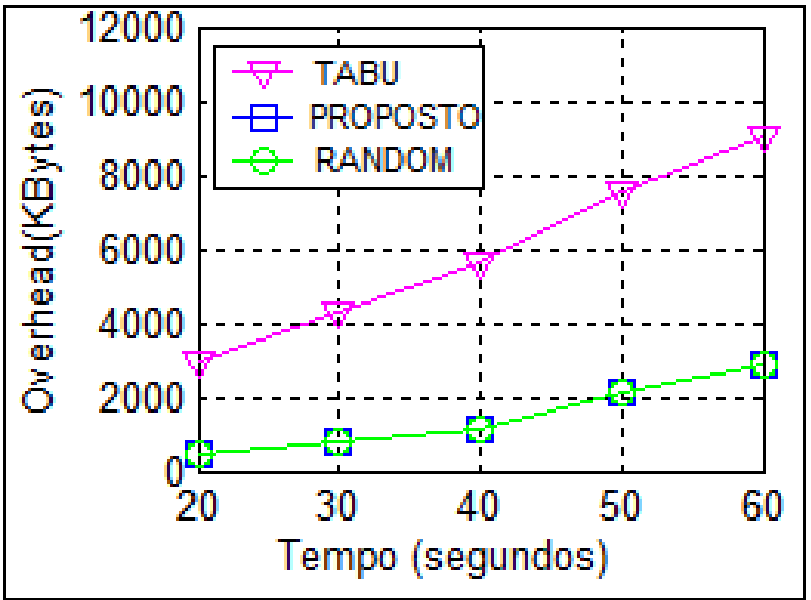

Figura 10: Overhead

A estratégia proposta apresenta atraso fim a fim próximo de TABU, com um incremento de $3,59 \%$ a $4,82 \%$ para um número de canais entre 2 e 4 . Comparado a RANDOM e SC, a estratégia proposta apresenta decréscimo de atraso fim a fim de $14,13 \%$ a $11,22 \%$ e de $39,10 \%$ a $18,05 \%$, respectivamente. Conforme o número de canais aumenta, o atraso fim a fim diminui devido a menor contenção entre os nós vizinhos a 2 saltos. O mais alto atraso fim a fim é apresentado por SC devido a este método apresentar a contenção máxima.

A Fig. 10 mostra o overhead no canal de controle variando com o tempo.

$\mathrm{O}$ overhead é medido em bytes devido aos diferentes tamanhos das mensagens de controle. TABU apresenta o mais alto overhead devido a ele trocar mensagens com todos os nós da rede. A estratégia proposta e RANDOM apresentam o mesmo overhead devido a eles usarem o mesmo mecanismo para a troca de mensagens, mudando somente o número do canal. Eles apresentam menor overhead que TABU devido a troca de mensagens serem limitadas a 2 saltos. No tempo de 40 segundos, o overhead aumenta devido as fontes de tráfego iniciarem a transmissão, aumentado a troca de mensagens de controle RTS/CTS. No gráfico, não é apresentado o método SC devido a ele não usar um canal de controle.

\section{Conclusões}

Neste trabalho foi apresentado uma estratégia de alocação de canais baseada em comportamento do nó para redes veiculares. A principal contribuição deste trabalho foi considerar algumas características do comportamento do nó, tais como a mobilidade, tráfego e popularidade para guiar o processo de alocação de canais. Foi utilizado uma abordagem distribuída para limitar a troca de mensagens na rede a 2 saltos, permitindo diminuir o overhead. As simulações mostraram que a estratégia apresentada resultou, quando comparado ao método RANDOM, em um ganho de vazão agregada de $9,2 \%$ a $12,61 \%$, com um ganho de taxa de entrega de pacotes médio de $11,65 \%$ e uma redução no atraso fim a fim da ordem de $14,13 \%$ a $11,22 \%$. Em relação ao método SC, o 
ganho apresentado pela estratégia proposta foi da ordem de $21,15 \%$ a $28,25 \%$, com um ganho na taxa de entrega de pacotes média de $17,18 \%$ e uma redução no atraso fim a fim na ordem de $39,10 \%$ a $18,05 \%$, conforme o número de canais aumenta. Os resultados da estratégia apresentada foram muito próximos da abordagem centralizada TABU, porém com menor overhead. Desta forma, a estratégia apresentada é mais indicada para situações em que a quantidade de nós é mais elevada, uma vez que, a rede é segmentada através dos canais e o overhead gerado é menor. Por fim, este trabalho abre caminho para pesquisas futuras tais como: a utilização de outros parâmetros de comportamento, utilização de qualidade de serviço e associação da estratégia de alocação de canais com protocolos de roteamento.

\section{Referências}

Aschenbruck, N., Ernst, R., Gerhards-Padilla, E. and Schwamborn, M. (2016). BonnMotion - A Mobility Scenario Generation and Analysis Tool. Available at https://sys.cs.uos.de/bonnmotion/doc/README.pdf (Accessed 3 April 2017).

Bharati, S. and Zhuang, W. (2018). Link-layer cooperative communication in vehicular networks, in S. I. Publishing (ed.), Wireless Networks, Springer International Publishing. Available at https://link.springer.com/content/pdf/bbm \% 3A978-3-319-58721-9\%2F1.pdf (Accessed 25 April 2019).

Chen, W. (2015). Vehicular communications and networks - architectures, protocols, operation and deployment, in W. Publishing (ed.), Vehicular Communications and Networks, Elsevier. Available at https: //www.sciencedirect.com/book/9781782422112/ vehicular-communications-and-networks\# book-description (Accessed 28 April 2019).

Chu, I., Chen, P. and Chen, W. (2012). An IEEE 802.11p based distributed channel assignment scheme considering emergency message dissemination, Proceedings of the 75th Vehicular Technology Conference, IEEE, Yokohama, Japão. Available at https://ieeexplore.ieee.org/document/6240201 (Accessed 15 April 2017).

Frigau, M. S. (2015). Social-based Forwarding in multi-channel Vehicular Networks, IEEE Symposium on Computers and Communication, IEEE, Larnaca, Cyprus. Available at https://ieeexplore.ieee.org/ document/7405511 (Accessed 2 Apr 2018).

Gupta, N., Prakash, A. and Tripathi, R. (2016). Clustering based cognitive mac protocol for channel allocation to prioritize safety message dissemination in vehicular ad-hoc network, Vehicular Communications 5(1): 44-54. https://www.sciencedirect.com/science/article/ $\mathrm{abs/pii/S2214209616300237.}$

Hande, R. S. and Muddana, A. (2016). Comprehensive survey on clustering-based efficient data dissemination algorithmsm for VANET, Proceedings of the International Conference on Signal Processing, Communication, Power and Embedded System,
IEEE, Paralakhemundi, India. Available at https://ieeexplore.ieee.org/document/7955516/ authors\#authors (Accessed 2 April 2018).

Hussain, S. A., Iqbal, M., Atif, S., Raza, I., Raza, H., Ali, A., Bashir, A. K. and Baig, A. (2017). An Efficient Channel Access Scheme for Vehicular Ad hoc Networks, Mobile Information Systems 2017: 110. https://doi.org/10.1155/2017/8246050.

Lemos, P. (2010). VANETs - Vehicular Ad hoc Networks. Available at https://ww.gta.ufrj.br/ensino/ eel879/trabalhos_vf_2010_2/lemos/index.html (Accessed 2 Feb 2019).

Lusheng, M., Djouani, K., van Wyk, B. J. and Hamam, Y. (2012). Evaluation and Enhancement of IEEE 802.11p Standard: A Survey, Mobile Computing 1(1): 15-30. https://archive.org/details/MC10022.

Mahdi, Z., A. K. M. Muzahidul, I., Nafees, M., Sabariah, B., , Ehab, M. M. and Seiichi, S. (2016). CMCS: a cross-layer mobilityaware MAC protocol for cognitive radio sensor networks, EURASIP Journal on Wireless Communications and Networking 2016: 1-15. https://jwcn-eurasipjournals. springeropen.com/ articles/10.1186/s13638-016-0652-y.

Nguyen, V., Kazmi, S. M. A. and Hong, C. S. (2015). Ecct: An efficient-cooperative adhoc mac for cluster-based tdma system in vanets, International Journal of Distributed Sensor Networks pp. 1-12. https://journals. sagepub.com/doi/full/ $10.1155 / 2015 / 746438$.

nsnam.org (2016). NS3 Manual. Available at https://www.nsnam.org/docs/manual/html/index. html (Accessed 15 Jan 2017).

Park, S., Chang, Y., Khan, F. and Copeland, J. A. (2013). Dynamic service-channels allocation (DSCA) in vehicular ad-hoc networks, Proceedings of the 1oth Consumer Communications and Networking Conference, IEEE, Las Vegas, USA. Available at https: //ieeexplore.ieee.org/document/6488469 (Accessed 18 June 2017).

Peng, H., BaoPing, Y., Zhi, L. and Limin, S. (2014). Cm-mac: A cluster-based multi-channel mac protocol for vanet, Journal of Computer Research and Development pp. 502-510. http://crad.ict.ac.cn/ EN/abstract/abstract2090.shtml.

Reddy, M. A. K. and Reddy, A. R. M. 1. (2017). PBMAC - Position Based Channel Allocation for Vehicular Ad hoc Networks, Journal of Advanced Networking and Applications 8: 3156-3160. http://www.ijana. in/papers/V8I4-5.pdf.

Rocha da Silva, M. and Rezende, J. F. (2006). A Dynamic Channel Allocation Mechanism for IEEE 802.11 Networks, VI International Telecommuniations Symposium pp. 1-6. https://www.gta.ufrj.br/ftp/ gta/TechReports/SiRe06a.pdf.

Sallem, Y., Bashir, A., Ahmed, E., Qadir, J. and Baig, A. (2012). Spectrum-aware dynamic channel assignment in cognitive radio networks, IEEE International Conference on Emerging Technologies, IEEE, Islamabad, Pakistan. Available at https: 
//ieeexplore.ieee.org/document/6375468 (Accessed 10 May 2018).

Subramanian, A. P., Gupta, M., Das, S. R. and Cao, J. (2008). Minimum interference channel assignment in multiradio wireless mesh networks, IEEE Transactions on Mobile Computing pp. 1459-1473. https://ieeexplore.ieee.org/ document/4509433/authors\#authors.

Sulistyo, S. and Alam, S. (2018). Performance study and optimization of multi-channel allocation in vanet under physical channel model, Wireless Networks pp. 1-13. https://link.springer.com/ article/10.1007/s11276-018-1770-8.

Tabassum, M., Razzaque, M. A., Hassan, M. M., Ahmad, A. and Alamri, A. (2016). Interferenceaware high-throughput channel allocation mechanism for cr-vanets, Eurasip Journal on Wireless Communications and Networking pp. 1-15. https://jwcn-eurasipjournals.springeropen.com/ articles/10.1186/s13638-015-0494-z.

Tomar, R. S. and Verma, S. (2010). RSU centric channel allocation in Vehicular Ad hoc Networks, Sixth International Conference on Wireless Communication and Sensor Networks, IEEE, Allahabad, India. Available at https: //ieeexplore.ieee.org/document/5712285 (Accessed 30 Oct 2017).

Zhao, T., Lu, S., Yan, W. and Li, X. (2014). A connectivity resilient dynamic multi-channel assignment method for vanet, Security and Communication Networks 8(10): 1855-1864. https://onlinelibrary.wiley.com/doi/full/10. 1002/sec. 1032. 